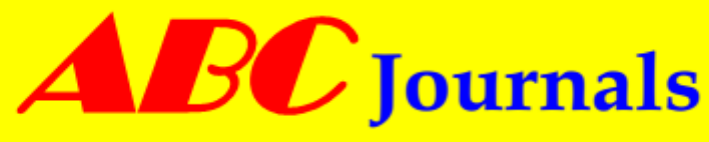

A new domain in research publishing

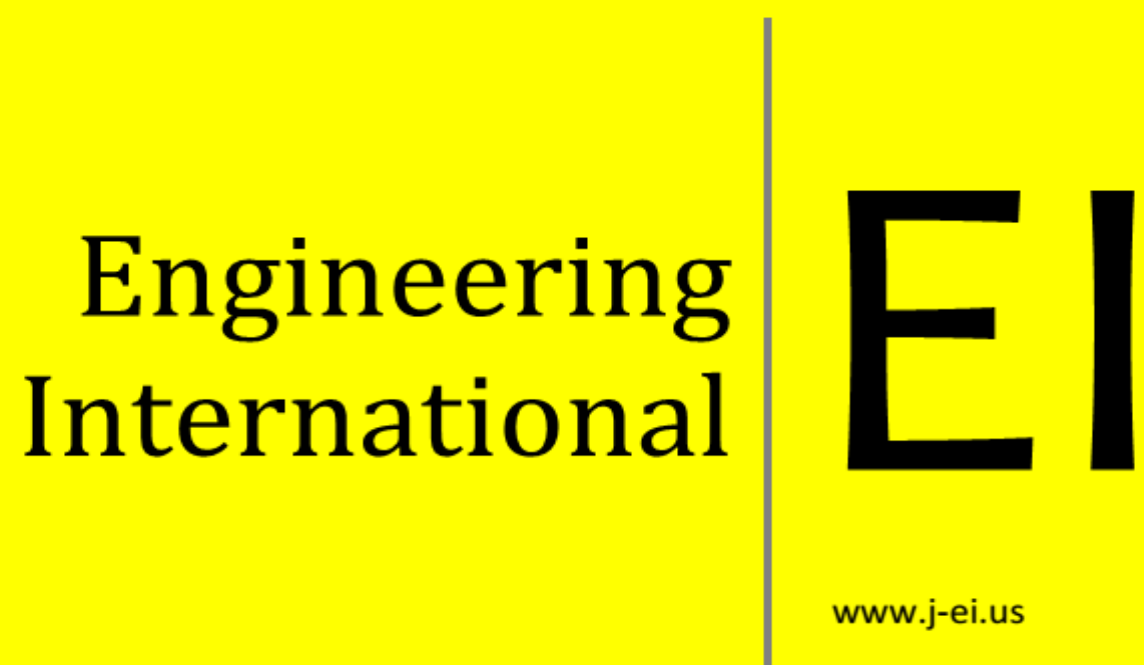

Asian Business Consortium 


\title{
Multipurpose Tactical Robot
}

\author{
Md. Taher-Uz-Zaman'1, Md. Sazzad Ahmed², Shabbir Hossain ${ }^{3}$, Shakhawat \\ Hossain $^{4}$, \& G. R. Ahmed Jamal ${ }^{5}$
}

1,2,3,4 Department of Electrical \& Electronic Engineering, University of Asia Pacific, Bangladesh

${ }^{5}$ Assistant Professor, Dept. of Electrical \& Electronic Engineering, University of Asia Pacific, Bangladesh

\begin{abstract}
This paper presents a general framework for planning a multipurpose robot which can be used in multiple fields (both civil and military). The framework shows the assembly of multiple sensors, mechanical arm, live video streaming and high range remote control and so on in a single robot. The planning problem is one of five fundamental challenges to the development of a real robotic system able to serve both purposes related to military and civil like live surveillance(both auto and manual), rescuing under natural disaster aftermath, firefighting, object picking, hazard like ignition, volatile gas detection, exploring underground mine or even terrestrial exploration. Each of the four other areas - hardware design, programming, controlling and artificial intelligence are also discussed.
\end{abstract}

Key words: Robotics, Artificial intelligence, Arduino, Survelience, Mechanical arm, Multi sensoring

\section{INTRODUCTION}

There are so many robots developed based on line follower, obstacle avoider, robotic arm, wireless controlled robot and so on. Most of them are built on the basis of a specific single function. For example a mechanical arm only can pick an object inside its reach. But what will happen if the object is out of its reach? Besides implementing a simple AI (e.g. line following, obstacle or edge detection) is more or less easy but that does not serve any human need. A.I. now-a-days are developing but yet its need time to make robot available for a civilian not only because of cost but also for reliability of A.I. Thus far, most of the militaristic robots are manual controlled like flying drone, unmanned vehicle (Okerea 2013). Because manual controls makes a robotic system more or less reliable and "command and be done". Even also communicating with a machine is most likely the important part of any robot. Means how it will behave when it needs to send any data to the user or down streaming data from user. Moreover using a robot outdoor is pretty a challenge because surface are not even as indoor. The work described in this paper is part of an effort to develop a largely integrated technology that will enable the design and implement of an robot that can be used not only in a war field but also in civil need, security and in a word capable of being used in different environment. Many issues need to be addressed before real robots that can be used in several fields. This paper considers most fundamental issues like planning, hardware design, programming and controlling to implement the project. All these issues were properly considered in constructing the proposed robot. 


\section{Motivation}

The results of research in this area will benefit a number of applications and have implication for several research areas within. Also it shows a possibility to make robotics familiar in civil field with military that the a single robot can be used for managing security purposes not only for surveillances but also on various ways and to save human lives beneath the ruin caused by environmental hazards like earthquake, cyclone, and hurricane.

\section{FundAMENTAL DESIGN ISSUES}

There are five fundamental issues involved in developing the robotic system for multipurpose robot: planning, hardware design, programming, controlling and artificial intelligence. A substantial amount of works need to be done in each of these areas.

\section{Planning}

As per described that our goal is to build a robot that can be served multi purposely both in military and civil, then it is essential to coordinating the substances that are in common for both civil and military. As the goal is make the robot all-rounder, several things are needed to be paid attention.

- Robotic system should be very sensitive to the environmental parameters like temperature, humidity, presence of volatile gas (e.g. LPG, Methane), CO, dense of oxygen, radioactivity and even the dew point. Because when robot will be used for exploring a cave or unknown underground mine all those parameters should be important. Also this function, specifically temperature and presence of LPG will become essential when robot will be used indoor security or home security.

- When robot will be used for home security, it should be concern about intrusion. Also home security should be autonomous. So, basic A. I. is inevitable for countering this situation. To safely surf inside a house and to provide anti-intrusion, system will need obstacle sensing, proximity sensing capability.

- Needless to say that, a Close circuit camera is must for any type of use related to rescuing, surveillances, exploring and so on. Besides Close Circuit camera should be wireless and night vision supported. No one expects sunlight inside an underground mine or cave. Also FPS rating of the camera may be brought under consideration because if robot is used to guard a military facility or an airport, it may need to live streaming a high velocity vehicle. If the FPS is below the minimal rating, then video or image can be distorted.

- When robot will be used to rescue people under ruin which unreachable to human mechanical arm will be vastly needed because robot may pick objects, remove light obstacle to make the road easier to move or may carry supplies to the victim in order to keep them alive a much longer until rescue team arrives.

- The robotic system should have a bi-directional voice communication system which can be interfaced by human as it is needed both in war field and house. The robot can be used as a "MOBILE COMMUNICATION BASE" via GSM network. Also it is needed during rescuing as victim under ruin may communicate with rescue team thorough the robot once it reaches to the victim.

- Selecting wheels are also a major factor about designing a multipurpose robot, because indoor and outdoor surfaces are not same. When robot will be used for rescuing or in an unknown terrain exploration, it will be difficult to move the robot efficiently if it designed only suitable for indoor. So it would be a challenging problem to find out the solution of wheeling system that is adaptive to all surfaces.

- $\quad$ Power is a great problem for robotics. Several functions can be added but it must be ensured that a proper power supply unit exists within the system. For example, when robot will be used for rescuing or exploring, battery power should be good enough to function the whole system properly for minimum $2 \sim 3$ hours. 
- Control unit should have a display unit, navigation unit with strong wireless system. Because exploring under ruin, underground mine is not same as just free surfing indoor.

\section{Hardware Design}

A good hardware design can increase the performance of the robot and often can make each of the other fundamental issues easier to deal with. First of all it is necessary to pick up the right material for robotic body and selecting necessary wheeling system.

Robotic wheeling system may take various shapes like tank type wheeling, two wheel type, four wheel type and six wheel type. Here it is prefer to set a three wheel type. But a two or three wheel type is not suitable over a good rescuer or surveyor robot because of its shape and bi-directional moving works. Four wheels are suitable for robot's easy moving on both surfaces like indoor smooth surface and outdoor rough ort uneven terrain. But gross weight of the total system must be a matter of concern, because it is directly related to the overall performance of the robot including the power reserve or battery. So nylon material made wheel and custom made tank tread are durable for surveying, rescuing and surfing rough terrain. A surveyor or rescuer robot not only just use wheel but also use wheel chain as like tank wheel chain robot which is simple, has good strength to carry load and also can run in harsh environment (Anjum 2012). It's very important to build up a suitable robotic body for easy, balanced and efficient moving and load carrying. For rescuing robot it is very important to create a shape of a tank type robot and body material should be very thin, high durability, electric shock protector, low weight, temperature and dielectric permittivity. The acrylic sheet is much suitable for that. It has much temperature and dielectric permittivity $(55.98 \mathrm{pF} / \mathrm{m})$ [12]. Here, robot body is built by acrylic sheet in a tank shape. Acrylic sheet's thickness and stretch depends on electrical breakdown strength. A thin sheet breakdown field scales as

$E_{b}=51 h^{-.25} \lambda^{.63}$.

As described earlier, multiple environmental parameters should be known for indoor security to terrains exploration. So, sensors should be chosen to sense parameters like humidity, temperature, gas (CO, LPG, and METHANE) etc. Also some reflective sensor should be used to measure parameters like distance, obstacle etc.

It is very much needed to see and control the robot from a control room or a hand help control unit if the robot is placed in a remote area. Live streaming video and audio system are needed in that case. Here, facility of live video update with $30 \mathrm{fps}$ for unlimited data streaming from a wireless range of about $1 \mathrm{Km}$ is added.

For a rescuer robot a mechanical arm is a crucial part to pick up something for rescuing or carrying a load. Here, the mechanical arm is constructed by three axes full servo motors, clipper and clampers. It is designed with high speed and accuracy in the three dimensional activities. The robotic arm, which is designed to have 5 degree freedom. But it has some problem to move freely when it works and takes command. So, it was converted into 4 degree freedom arm. It is observed that shoulder variability is higher than other joint angles in repeated movements. It is also considered that only one robotic arm is not good enough, rather two arms are needed to do human like work. One hand is specially made for slippery objects holding and other is specially made for rough objects holding. Here it is also considered the co operating task of hybrid scheme of two-arm robot. It has a unique system workspace coordinate of vectors. It is also consider the joint spaces and kinematics and statics. The arm control has two level of control hierarchy is needed. Here it needed also variable structure controllers. A good system programming and implementation is needed. Decentralization of structure is important for that purpose (Ozguner 1987). The improvement to robot arm limb section is also important. Otherwise it cannot take a rough object easily. Here motion control of two arm robot is effectively needed. A high range 
multiple arm lower or upper limb robots are needed to synchronized movement of the two robotic arms. It contributes in overcoming the instabilities caused by interference of two separate controls (Jurak 2007). Wireless video transmission in rescuer or surveyor portion is needed. Here the Rx and Tx distance is 200 meter. Here working of a wireless transmitting and receiving technology working at an individual frequency of its own. Different Communication channel frequency is used for different purposes. For military use, different frequency will be needed.
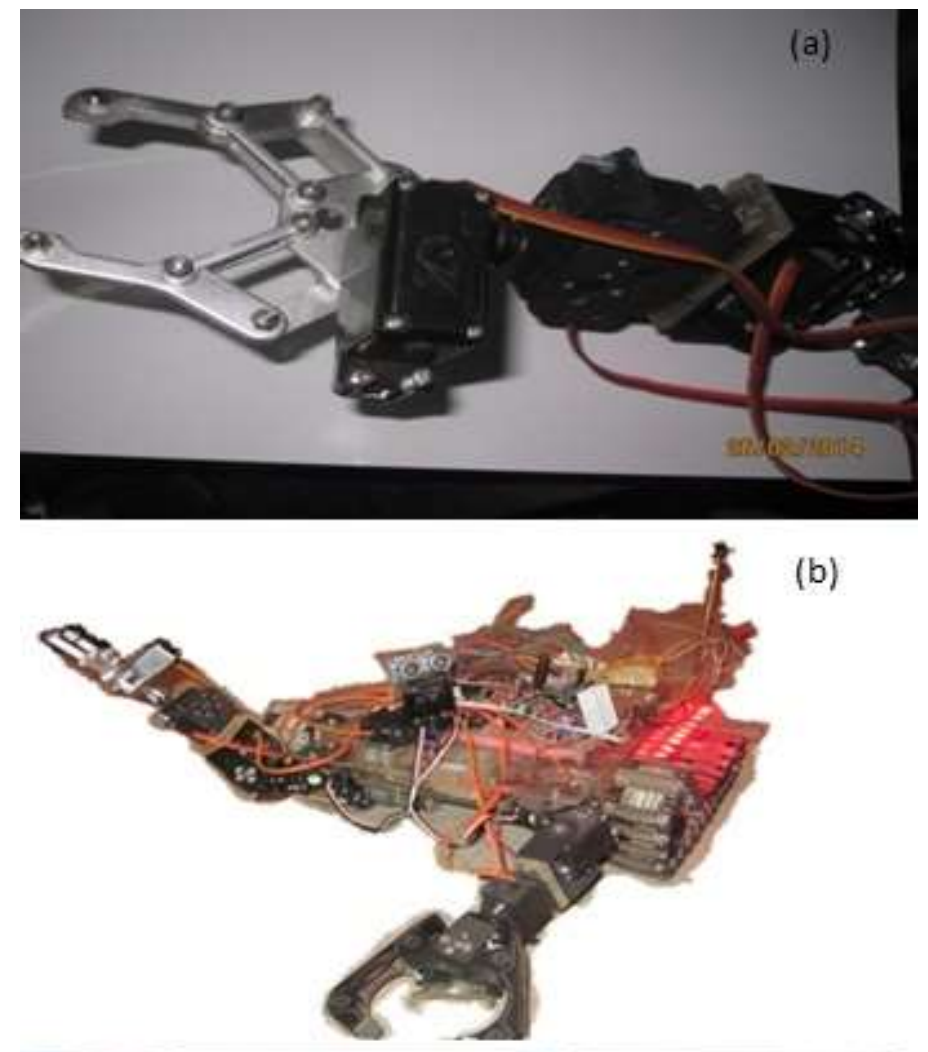

(b)

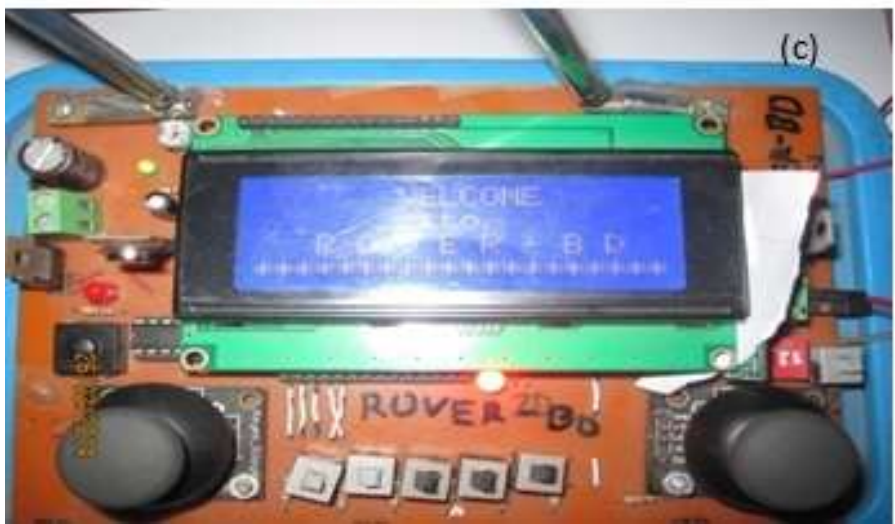

Figure 1 Construction of the proposed robot. (a) Mechanical arm (b) full body of the robot and $\mid$ (c) Control and Display unit. 
This robot can also work at an individual frequency. Sometimes in transmission the quality of receiving video is a problem. It may cause by wireless channel fading, Doppler phenomenon and also for limited bandwidth (Duan 2009). Here video transmission needs extra energy consumption from battery. So, it followed lower power consumption video transmission policy.

Power supply is the vital part of hardware design of robot, because whole plan and design depends on power system. Here power system is provided from a mobile power system. As a rescuer and surveyor robot, it cannot be operated from a cable power supply. So, it needs a light weight and portable battery. Required battery capacity is proportional to weight of the robot and weight is inversely proportional to the efficiency of the robot. Now days, an energy efficient lithium polymer (Li-po)battery are used for extra power giving which has very less weight with high capacity. Important parts of the body of the robot are shown in Fig.1 (a), (b) and (c).

When the rescuer robot need to go out of its normal range from transmitter, then GSM technology is needed for its control and communication. It can let user to be informed by message or mobile calling or mobile video calling with its high data transfer rate (3G) technology. By that a user can easily command robot back what have to do now. It can also give the receiving display panel if there has any gas leak, fire alarm etc. It also works in adaptive surveillance (Ramu 2008).

\section{Programming:}

All Robotic works depends on a systematic simulation. For that there needs a microcontroller to do some programming. Here an Arduino Uno (Atmega328) microcontroller is used. It is based on java programming. Here $\mathrm{C}++$ programming also included. It can operate all type of hardware by programming such as DC motor, servo motor, various types of sensors, GSM module (Araujo 2013). It is programmed by Arduino Uno software. The advantage of this software's is it is very reliable and easy to burn the microcontroller. Our robot is fully controlled from that microcontroller.

\section{Controlling:}

Controlling mechanism of the Robot influence its surveillance or rescuing operation. Effective controlling is the main demand for surveillance. Our robot is controlled from a hand held control panel. It also uses a LCD display to get updates. Here one can see the display of sensor system outputs, video output and alarming output. When a user send data wirelessly to the robot, only one frequency cannot cover all those command at a time. So, here we used two frequencies. One frequency is used for robot arm and camera (movement and data sending) and other frequency is used for movement of mechanical arm and robot body. It can be noted that, though robot body and mechanical arm are operated by same frequency but they need not to always move at a time as they are controlled by different switch in the control panel.

\section{Artificial Intelligence:}

Artificial intelligence is required for any robot, especially for rescuer robot or surveillance robot. Our robot can control itself in automatic mood using some artificial intelligence that we provided to it. While in Artificial intelligence mode and face any adverse situation, it can automatically start its GSM module on and send data to the user. 180 degree rotating camera also gives video update to the user. Its ultrasonic device can also measure the distance automatically and can automatically decide how to select its moving path or direction. It can take decision to compare two place or more distance and then it can take self-decision where it can go or which direction it should avoid. 


\section{TEST AND OPERATION}

We constructed our robot maintaining all these design criteria mentioned so far and tested its operation. It was operated quite successfully and performed all the works for which it was designed. Its two mechanical arms can grip two types of object. One is slippery type object and other is rough object. A good surveillance output was observed and sensory system worked well. All Control commands worked successfully and communicating efficiency were high.

Beside the success, we also observed some problems with its design. The chain of the wheels was observed to slip time to time. The problem was solved by adding a metal chain which strongly holds the wheel. A plastic type rough material was also placed outside it which helps the body as well as robot not to slip while running in a sloppy area. There are some future up gradation plans for further improvement of this robot. Light weight solar panel will be placed around its body so that it can take power from sunlight during day time as well as can charge its battery. Besides, installation of fingerprint protected system in its control unit, hidden multiple electric metal plates for anti-theft system, computer based navigation system, Radioactive chemical detection, Water proof caching system, Self-destruction system in case of captured by hostile personnel etc are also included in its future up gradation plan.

\section{CONCLUSION}

A general framework for planning a multipurpose robot is discussed which can be used in multiple fields (both civil and military). The framework shows the assembly of multiple sensors, mechanical arm, live video streaming and high range remote control and so on in a single robot. For civil use, it can detect gas leak in home or industry, surveillance of shopping mall, parking lot, office, industry, bank, museums, rescuing operation during natural disaster and aftermath like searching for human, supplying food and other essentials to the people under unreachable ruin. It can also perform military surveillance like spying enemy base, exploring unknown enemy territory, detecting explosive mine underneath surface, night security on various spheres, explosive disposal, auto-pilot enabled security and anti-intrusion defence, as a mobile communication base during war, natural disaster, GSM communication from any location. Its future improvement will enhance its capability to do some other works also.

\section{Reference}

A. Aboshosha, A. Zell, "Adaptation of rescue robot behavior in unknown terrains based on stochastic and fuzzy logic approaches", Proc. of IEEE/RSJ International Conference on Intelligent Robots and Systems,2003(IROS 2003), Vol. 3, pp.02859-2864, 27-31 Oct, 2003.

Anjum,s. "Design of non-conventional chain drive mechanism for a mini-robot", Proc. of 2012 International conference on robotics and artificial intelligence(ICRAI), pp. 103-107, 22-23 Oct, 2012.

Araujo.A, "Integrating Arduino-based educational mobile robots in ROS", 2013 13th international conference on Autonomous robot systems(robotica), pp. 1-6, 22-24 Apr, 2013.

D.T. Okerea, U.Diala, N.Onuekwusi, L.O. Uzoechi, G.Chukwudebe,"Improving security and emergency response through the use of unmanned vehicles" IEEE International Conference on Emerging \& Sustainable Technologies for Power \& ICT in a Developing Society (NIGERCON)-2013, pp.263-269, 14-16 Nov, 2013.

Duan dagao, "A robust error resilience Scheme for wireless video transmission", Proceeding of 2nd international conference on computer science and information technology (ICCSIT) , pp. 217221, 8-11 Aug, China, 2009. 
F.D.Makaya, D.Chatelain, L.W.Snyman,"Design and performance assessment of a prototype wireless controlled robot.", Proc. Of 12th International Symposium on Electron Devices for Microwave and Optoelectronic Applications 2004, pp.115-118, 8-9 Nov, 2004.

Hiu Liu, N.Stoll, S. junginger, K.Thurow, "A common wireless remote control system for mobile robots in laboratory", Proc. of 2012 IEEE International Instrumentation and Mesurement Technology Conference (I2MTC), pp. 688-693, 13-16 May, 2012.

I. S. Jacobs and C. P. Bean, "Fine particles, thin films and exchange anisotropy in Magnetism, vol. III, G. T. Rado and H. Suhl, Eds. New York: Academic, pp. 271-350, 1963.

Juangshui Huang, "The thickness and stretch dependence of the electrical breakdown strength of an acrylic dielectric elastomer", Appl. Phys. lett., Vol. 101, Issue 12, 122905, 2012.

Jurak,M. "An approach to consider upper limb kinematics for the Improvement of Motion control in a two arm robotic rehabilitation system." IEEE 10th international conference on Rehabitation robotics ICORR-2007, 99.274-282, 13-15 June, 2007.

P. Krasnansky,F.Toth,V.V Huertas, B.Rohal'llkiv, "Basic labrotary experiments with an educational robotic arm", Proc. Of International conference on Process Control(PC), pp. 510-515, 18-21 june, 2013.

Qiao Liu,Yong-gang Lu, Cun-xi Xie, "Optimal Genetic Fuzzy Obstacle Avoidance Controller of Autonomous Mobile Robot Based On Ultrasonic Sensors" , Proc. Of IEEE International Conferenc on Robotics and Biomimetics, pp. 125-129, 17-20 Dec, 2006.

R. Kunanuruksapong, "Effects of temperature and dielectric permittivity on electrorheological properties of elastomers", Proc. of 7th IEEE Conf on nanotechnology-2007, pp.1081-1084, 2-5 Aug, 2007.

Ramu,G.V, "Remote robot training of its artificial neural network through GSM communication", Proc. of 3rd international conference on systems and networks communications- 2008,pp 355358, 26-31 Oct, 2008.

S. Kobayashi, K. Nonaka ,"Real-time optimized obostacle avoidance for robotic vehicles: Indoor experiments", ICCAS-SICE, pp. 3193-3198, 18-21 Aug, 2009.

S.E.M. Bajestani, A. Vosoughinia, "Technical report of building a line follower robot", Proc. Of International Conference On Electronics and Information Engineering (ICEIE), Vol. 1, 2010,

T. Yali, "Three axes full servo robotics design and application", Proc. of 2010 international conference on computer application and system modeling (ICCASM), Vol. 13, pp.142-146, 22-24 oct, 2010.

Tao kang, "Determining natural arm configuration along reaching trajectory", 25th conference of IEEEengineering in medicine and biology society-2003, Vol. 2, pp 352-361, 2005.

U. Ozguner, , "Decentralized variable structure control of a two-arm robotic system", Proc. of 1987 IEEE international conference on robotics and auto mation., Vol. 4, pp. 1248-1254, 1987.

Uchiyama,M, "Hybrid position/force control for coordination of a two-arm robot", Proc. of 1987.IEEE international conference on robotics and automation. Vol. 4, pp1242-1247, 1987.

Vishnu R.Kale,V.A.Kulkarni, “Object Sorting System Using Robotic Arm”. International Journal of Advanced Research in Electrical, Electronics.and.instrumentation.Vol 2,Issue 7, July, 2013. 